\title{
Maxillary protraction in adult cleft lip and palate by a rigid external distraction device with dentoskeletal anchorage
}

\author{
Bengisu Akarsu' \\ Tulin Taner ${ }^{1}$ \\ Gokhan Tuncbilek ${ }^{2}$ \\ M. Emin Mavili²
}

\section{ABSTRACT}

Objective: The objective is to evaluate the effects of maxillary distraction osteogenesis (DO) in an adult patient with unilateral cleft lip and palate (UCLP) by using a rigid external distraction (RED) device with dentoskeletal anchorage.

Method: 31-year-old male patient with UCLP with severe maxillary hypoplasia, dolichofacial growth pattern, negative overjet and $1.5 \mathrm{~mm}$ openbite. After pre-surgical orthodontic treatment, an intra-oral appliance was modified to prevent extrusion of the molars and clockwise rotation of the mandible. Stainless steel plates were soldered bilaterally to the intra oral appliance at the level of canines. During surgery, miniplates were inserted in the maxillary segment and fixed to the plates of the intra oral appliance with screws.

Results: The mean distraction length was $12 \mathrm{~mm}$ immediately after DO. SNA increased from 730 to 820 after distraction. A significant advancement of the maxilla and correction of the sagittal Class III skeletal relationship was achieved. The vertical position of the mandible and the face was kept stable, and the soft tissue profile became more balanced.

Conclusion: This intra oral appliance design achieved desired skeletal changes during maxillary protraction with RED device in dolichofacial CLP patient. Occlusion and facial profile changes was found to be stable in 1-year follow-up. (Eur J Dent 2012;6:206-211)

Key words: Orthodontics; cleft lip; external distraction

Department of Orthodontics, Faculty of Dentistry, Hacettepe University, Ankara, TURKIYE

2 Department of Plastic and Reconstructive Surgery, Faculty of Medicine, Hacettepe University, Ankara, TURKIYE

- Corresponding author: Dr. Bengisu Akarsu Hacettepe Universitesi, Dishekimligi Fakultesi, Ortodonti AD., 06100 Sihhiye, Ankara TURKIYE Tel: +903123052290 Fax: +903123091138

Email: bengisuakarsudyahoo.com

\section{INTRODUCTION}

Distraction osteogenesis (DO) of the maxilla appears to be superior to conventional orthognathic surgery in cleft lip and palate (CLP) patients with severe maxillary deficiency. ${ }^{1}$ The Rigid External Distraction (RED) system provides gradual distraction of the maxilla and can change the vertical and horizontal vectors of distraction. The RED device requires a rigid intra oral appliance to apply 
traction through the dentition to the entire maxilla. The number of teeth is important for the rigidity of the appliance. ${ }^{2}$ In the absence of sufficient tooth support, unfavorable maxillary posterior teeth extrusions may occur instead of desired skeletal changes. This leads to unsatisfactory aesthetic results in dolichofacial CLP patients. The aim of the present case report was to evaluate the effects of maxillary distraction osteogenesis in a dolichofacial adult patient with CLP by using a RED device with dentoskeletal anchorage.

\section{CASE REPORT}

A 31 -year-old male patient with unilateral CLP presented a concave profile due to severe maxillary hypoplasia. The patient exhibited an Angle Class-III malocclusion with circular crossbite between the upper second molars and an anterior openbite (Figure 1 [a-e]). Maxillary right and left laterals were congenitally missing. Maxillary right and left central teeth, the maxillary right canine, the maxillary right and left first molars and the mandibular right first molar had profound caries. There was also an apical lesion on the mesial root of the mandibular right first molar. Cephalometric analysis revealed that the patient had a skeletal Class-III relationship, severe maxillary hypoplasia, a dolichofacial growth pattern, and palatally inclined upper incisors (Table 1). The treatment plan included maxillary advancement through the use of distraction osteogenesis after orthodontic treatment.

\section{Treatment Progress}

Maxillary right central, right and left first molars were extracted. The mandibular right first molar underwent endodontic treatment before the initiation of orthodontic treatment. The orthodontic treatment was initiated with a quadhelix appliance to expand the upper arch. Before bonding the upper teeth, the maxillary right canine and left central canine also underwent endodontic treatment; then prosthetic restorations were performed. Endodontic treatment of the mandibular right first molar was not successful, so this tooth was extracted and the space was maintained. During leveling and alignment, the patient could not maintain a high level of oral hygiene. Therefore, the mandibular right second and left first molar underwent endodontic treatment. After the inser- tion of $0.016 \times 0.022$ stainless steel archwires (Figure 2 [a-e]), the intra oral appliance was prepared with a headgear facebow for distraction. The intra oral appliance was modified by soldering stainless steel plates with a size of $10 \times 10 \mathrm{~mm}$ and a thickness of $1 \mathrm{~mm}$ at the level of the canines, bilaterally (Figure 3). The intra oral appliance was cemented to the upper molars, and the transpalatal bar and inner bow were connected with ligature wires through the embrasures between the teeth. During the surgery, miniplates were inserted into the maxillary segment and fixed to the plates of the intra oral appliance with screws.

\section{Surgery and Distraction Protocol}

A complete Le Fort I osteotomy was performed. After the maxillary osteotomy was completed, the halo portion of the RED device (Martin KLS, Germanyl was fixed around the head with three scalp screws on each side. During the surgery, miniplates were inserted into the maxillary segment and fixed to the plates of the intra oral appliance with screws (Figure 3). After a latency period of 3 days, the maxilla was distracted at a rate of $1 \mathrm{~mm}$ per day for 2 weeks.

Once the appropriate amount of distraction was achieved, the RED system was left in place for 4 weeks. After the consolidation period, the RED device was removed, and the patient was told to use an orthodontic face mask for a retention period of 8 weeks. Orthodontic treatment was completed 8 months after the surgery (Figure 4 [a-e]). The patient was referred for prosthetic treatment and a removable acrylic partial denture with a metal base was placed (Figure 5 [a-e]).

\section{RESULTS}

Favorable occlusion with an acceptable incisor relationship was achieved. Significant advancement of the maxilla and correction of the Class III skeletal relationship were achieved. The patient's soft-tissue profile became more balanced.

The amount of maxillary advancement was $12 \mathrm{~mm}$ after distraction. The SNA angle changed from $73^{\circ}$ to $82^{\circ}$ at the end of the treatment, and the facial axis remained stable (Table 1). The superimposition of pre- and post-treatment cephalometric radiographs is shown in Figure 7.

The one-year post-treatment occlusion and facial profile were stable (Figure 6 [a-e]). Compari- 


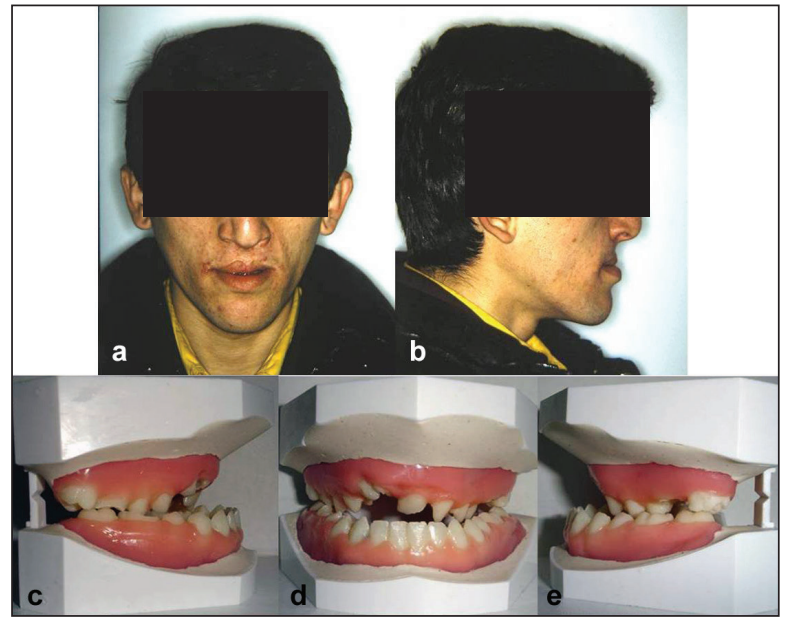

Figure 1. Pre-treatment extra oral photographs (a-b) and dental cast model (c-e) of the patient.

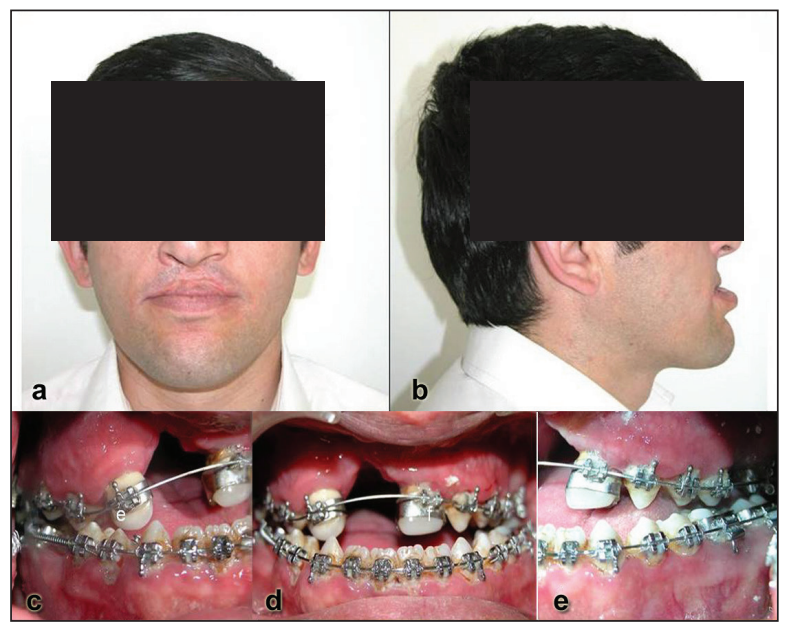

Figure 2. Pre-surgical extra oral (a-b) and intra oral (c-e) photographs of the patient.

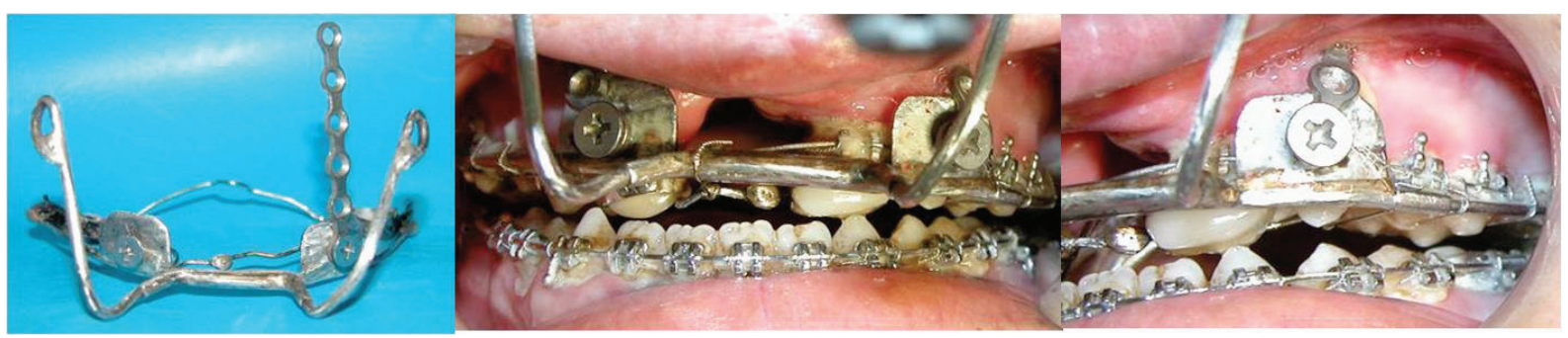

Figure 3. Modified intra oral appliance of the RED system.

Table 1. Cephalometric measurements at T1, T2, T3 and T4 (T1:pre-treatment, T2: pre-surgical, T3: post-treatment, T4: 1 year of post-treatment).

\begin{tabular}{|c|c|c|c|c|}
\hline Cephalometric analysis & $\mathrm{T} 1$ & $\mathrm{~T} 2$ & T3 & $\mathrm{T} 4$ \\
\hline $\mathrm{SNA}^{\circ}$ & 73 & 73 & 82 & 81 \\
\hline $\mathrm{SNB}^{\circ}$ & 79 & 78 & 78 & 78 \\
\hline $\mathrm{ANB}^{\circ}$ & -6 & -5 & 4 & 3 \\
\hline Maxillary depth ${ }^{\circ}$ & 85 & 83 & 91 & 90 \\
\hline Maxillary height ${ }^{\circ}$ & 70 & 73 & 72 & 73 \\
\hline Convexity $^{\circ}$ & -11 & -12 & 0 & 0 \\
\hline Facial depth ${ }^{\circ}$ & 93 & 91 & 91 & 90 \\
\hline Facial axis ${ }^{\circ}$ & 87 & 84 & 84 & 82 \\
\hline $\mathrm{FMA}^{\circ}$ & 29 & 33 & 32 & 33 \\
\hline GoGnSN ${ }^{\circ}$ & 41,5 & 44 & 42 & 42 \\
\hline Y axis ${ }^{\circ}$ & 60 & 62 & 62 & 63 \\
\hline Lower facial height $\mathrm{mm}$ & 48 & 52 & 51 & 52 \\
\hline U1-NA。 & -3 & 14 & 17 & 15 \\
\hline U1-NA mm & -4 & 1 & 1 & 1 \\
\hline U1-SN ${ }^{\circ}$ & 71 & 85 & 90 & 91 \\
\hline $\mathrm{U} 1-\mathrm{FH}^{\circ}$ & 83 & 96 & 100 & 101 \\
\hline L1-NB 。 & 14 & 17 & 18 & 17 \\
\hline L1-NB mm & 3 & 4 & 4 & 5 \\
\hline IMPA。 & 74 & 76 & 78 & 76 \\
\hline FMIA ${ }^{\circ}$ & 77 & 72 & 70 & 71 \\
\hline Overbite $\mathrm{mm}$ & $-1,5$ & $-1,5$ & $-0,5$ & 0,5 \\
\hline Overjet mm & -16 & -13 & 1 & 0,5 \\
\hline Lower lip-E plane mm & $-0,5$ & 0,5 & $-3,5$ & -2 \\
\hline Upper lip-E plane mm & $-14,5$ & $-12,5$ & $-6,5$ & -6 \\
\hline
\end{tabular}

European Journal of Dentistry 


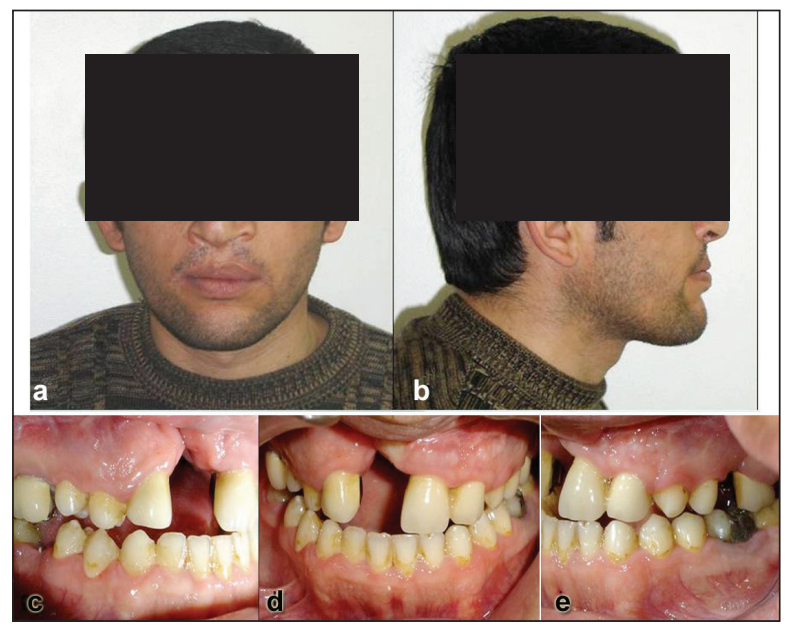

Figure 4. Post-treatment extra oral (a-b) and intra oral (c-e) photographs of the patient.

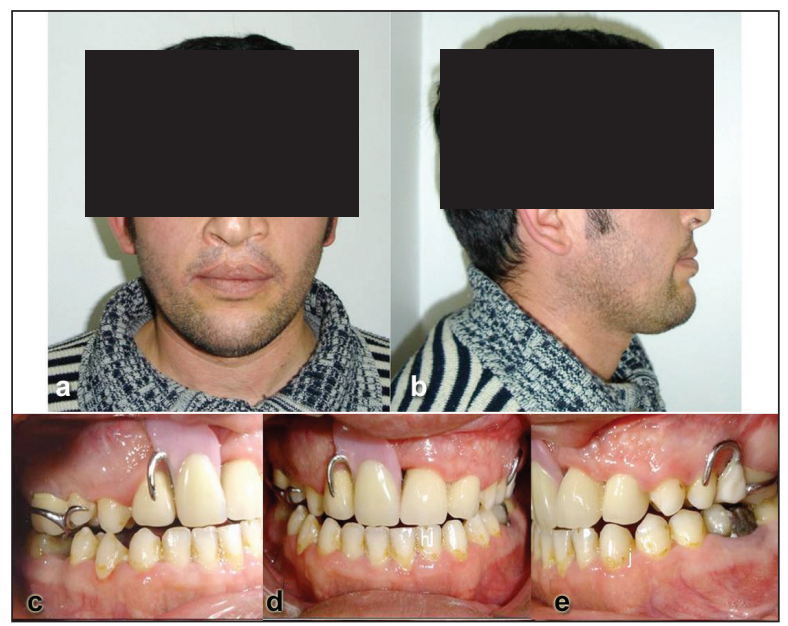

Figure 5. Extra oral (a-b) and intra oral (c-d) photographs of the patient after prosthetic treatment.

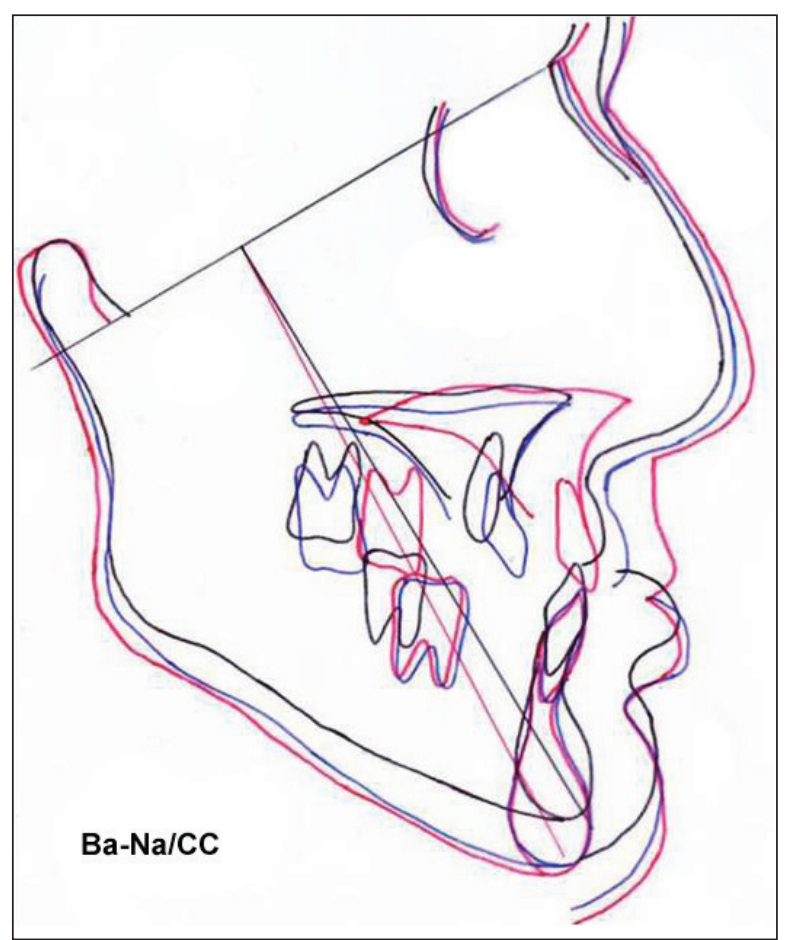

Figure 7. Cephalometric superimposition (black line: initial, blue line: pre-surgical, red line: final).

April 2012 - Vol.6 son of the final and 1-year post-treatment cephalograms showed minimal changes in the skeletal pattern (Figure 8 and Table 1): the maxilla had moved $1 \mathrm{~mm}$ backward, the mandible had moved downward slightly, the maxillary molars had settled downward, there was a slight reduction in the inclinations of the mandibular and the maxillary incisors. A small amount of increase in lower-lip fullness was found.

\section{DISCUSSION}

A considerable amount of maxillary advancement was made possible without a vertical change in facial dimensions by using a new intra oral ap-

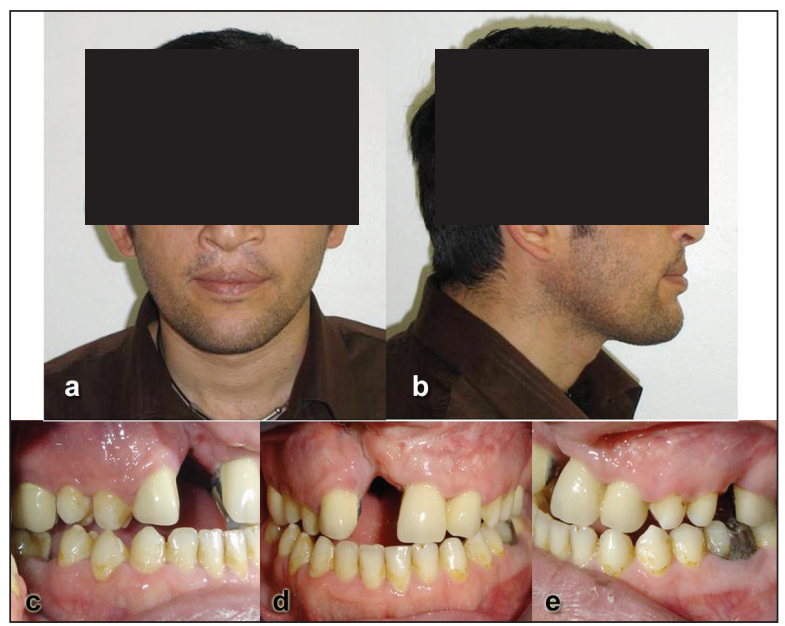

Figure 6. Extra oral (a-b) and intra oral ( $c-d)$ photographs of the patient after 1-year of post-treatment.

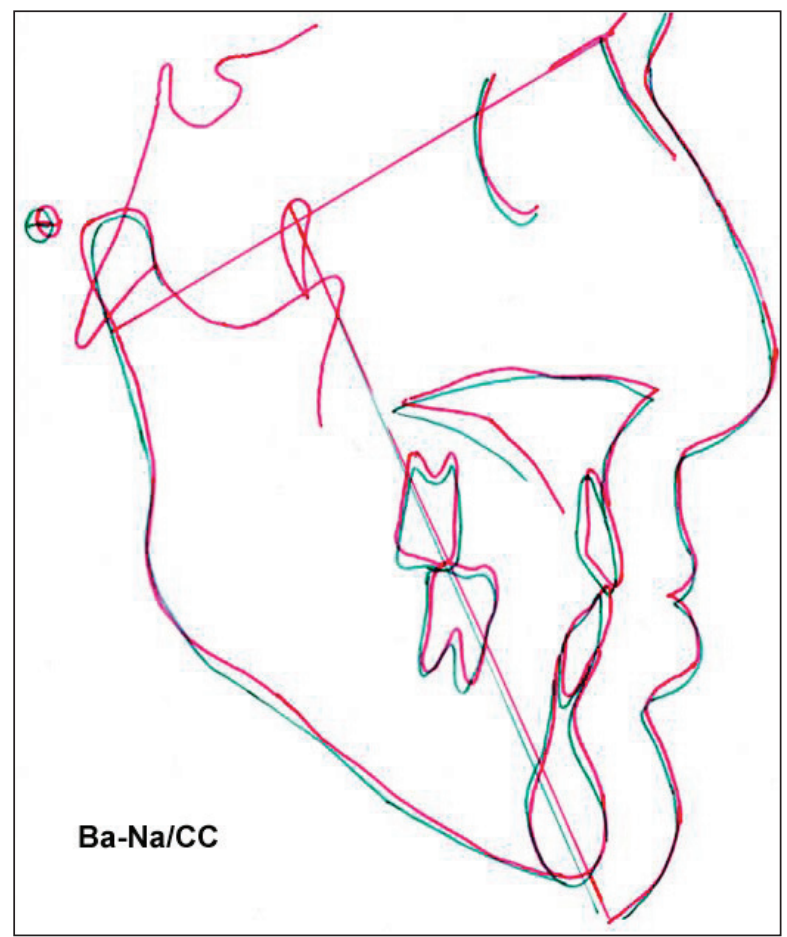

Figure 8. Cephalometric superimposition (red line: final, green line: 1-year posttreatment). 
pliance design in this dolichofacial CLP patient who had lost multiple teeth.

Advancement of the maxilla is more difficult to treat with conventional surgical and orthodontic approaches in patients with cleft lip and palate than in noncleft patients because the required amount of maxillary advancement is generally larger in cleft patients; in addition, these patients often have thin or structurally weak bone, absent and aberrant dentition, or scarring of the palatal, pharyngeal and facial soft tissues.

Conventional surgical treatment that includes Le Fort I maxillary advancement in adult cleft lip and palate cases with severe maxillary deficiency has an increased skeletal and occlusal relapse tendency because of severe scarring. ${ }^{3}$ Another method for correcting significant maxillary deficiencies may be a two-jaw approach. ${ }^{4}$ With this technique, occlusion can be corrected, but the maxilla remains deficient. Maxillary distraction osteogenesis with a RED device provides an attractive alternative for the treatment of CLP patients with maxillary deficiency. Maxillary distraction osteogenesis with a RED device has proven to be more effective in improving the soft-tissue profile of the cleft-lip-palate patients than conventional maxillary advancement. 4,5 There are several advantages of the use of distraction osteogenesis Advancement of the maxilla slowly into the desired occlusion is achieved and this allows for extensive advancement and overcomes soft-tissue tension. Compared with conventional Le Fort I osteotomy, distraction osteogenesis facilitates greater protraction distances. With the RED device, adjustment of the vectors of distraction is possible at any time during the distraction process, and the osteotomy design meets aesthetic requirements. ${ }^{1}$ In the present case, a high Le Fort I osteotomy was performed to allow maximal correction of the patient's facial profile because maxillary hypoplasia was observed not only in dentoalveolar region but also in the malar regions.

Our patient had congenitally missing maxillary laterals, five teeth with profound caries and one tooth with an apical lesion, which was treated endodontically. Four of these teeth were extracted because of the associated poor prognosis. It would therefore be difficult to maintain the anchorage of the intraoral appliance and to control the forces that were to be directly transmitted to the upper molars. Thus, the rigidity of the intraoral appliance was enhanced with miniplates connected to the anterior portion of the maxilla. Based on the literature, multiple circumdental wires have been used to create a rigid appliance to transmit the distraction forces to various teeth. ${ }^{1}$ In this case report, an RED device with a dentoskeletal anchorage was introduced to increase the rigidity of the intra oral appliance during traction. The intra oral part of the appliance was fabricated from a headgear face-bow to apply traction to the maxilla through the dentition. Dentoskeletal anchorage was obtained through the stainless steel plates soldered to the face-bow at the level of the canines, bilaterally. During the surgery, screws were fixed to the miniplates and inserted into the maxilla.

Temporary skeletal anchorage devices have been presented for the orthopedic correction of skeletal problems, ${ }^{6}$ and miniplates are considered to be effective anchorage devices. ${ }^{7}$ De Clerck et al ${ }^{6}$ achieved orthopedic traction of the maxilla with maxillomandibular elastics between miniplates placed on the maxilla and mandible in young maxillary-deficient patients. The ideal age and the amount of force for this type of traction remain to be determined. When performed with the RED system, skeletal anchorage for maxillary distraction osteogenesis has the advantage of transferring the distraction forces directly to the bone. ${ }^{8}$

The described intra oral appliance for the RED system is not intended to replace skeletal anchorage techniques. It offers an alternative to restrict the dentoalveolar movement during maxillary distraction in CLP patients, especially when the patient has lost multiple teeth and has a dolichofacial growth pattern.

This new intra oral appliance design enabled us to obtain the desired skeletal changes during maxillary protraction with the RED device in a dolichofacial CLP patient who had lost multiple teeth. Occlusion and facial profile changes were found to be stable at the 1-year follow-up.

Acknowledgement

We would like to thank Dr. Sencam Suicmez for prosthetic rehabilitation of the patient. 


\section{REFERENCES}

1. Polley JW, Figueroa AA. Rigid external distraction: its application in cleft maxillary deformities. Plast Reconstr Surg 1998; 102:1360-1372.

2. Kitai N, Kawasaki K, Yasuda Y, Kogo M, Murakami S, Kreiborg S, Takada K. Rigid external distraction osteogenesis for a patient with maxillary hypoplasia and oligodontia. Cleft Palate Craniofac J 2003;40:207-213.

3. Welch TB. Stability in the correction of dentofacial deformities: a comprehensive review. J Oral Maxillofac Surg 1989;47:1142-1149.

4. Harada K, Baba Y, Ohyama K, Omura K. Soft tissue profile changes of the midface in patients with cleft lip and palate following maxillary distraction osteogenesis: a preliminary study. Oral Surg Oral Med Oral Pathol Oral Radiol Endod 2002;94:673-677.

5. Wen-Ching Ko E, Figueroa AA, Polley JW. Soft tissue profile changes after maxillary advancement with distraction osteogenesis by use of a rigid external distraction device: a 1-year follow-up. J Oral Maxillofac Surg 2000;58:959-969.

6. De Clerck HJ, Cornelis MA, Cevidanes LH, Heymann GC, Tulloch CJ. Orthopedic traction of the maxilla with miniplates: a new perspective for treatment of midface deficiency. J Oral Maxillofac Surg 2009;67:2123-2129.

7. Cornelis MA, Scheffler NR, Mahy P, Siciliano S, De Clerck HJ, Tulloch JF. Modified miniplates for temporary skeletal anchorage in orthodontics: placement and removal surgeries. J Oral Maxillofac Surg 2008;66:1439-1445.

8. Minami K, Mori Y, Tae-Geon K, Shimizu H, Ohtani M, Yura Y. Maxillary distraction osteogenesis in cleft lip and palate patients with skeletal anchorage. Cleft Palate Craniofac $J$ 2007;44:137-141. 